Case Report

\title{
Post traumatic ureteral defect brigded with ileal segment (Yang-Monti) for inferior ureterocalycostomy: a rare case report with review of literature
}

\author{
Debanga Sarma, Rajeev T. P., Ashish Ghanghoria*, Sasanka Kumar Barua, \\ Puskal Kumar Bagchi, Mandeep Phukan
}

Department of Urology, Gauhati Medical College and Hospital, Guwahati, Assam, India

Received: 24 December 2019

Accepted: 01 February 2020

\section{*Correspondence:}

Dr. Ashish Ghanghoria,

E-mail: ashishghanghoria0311@gmail.com

Copyright: ( ) the author(s), publisher and licensee Medip Academy. This is an open-access article distributed under the terms of the Creative Commons Attribution Non-Commercial License, which permits unrestricted non-commercial use, distribution, and reproduction in any medium, provided the original work is properly cited.

\begin{abstract}
Ureteric substitution using the Yang-Monti principle was reported as a modification of simple ileal ureter replacement. A patient underwent ileal ureteral substitution using a reconfigured ileal segment of Yang-Monti principle in our clinical centre. Authors report a case of a 41year old male involved in a homicidal stab injury with isolated renal pelvis injury underwent Thompson's renal capsular flap repair. After 6 weeks, double J (DJ) stent was removed and following that patient developed urinoma. Percutaneous drain was placed to drain urinoma on emergency basis and again 6 Fr DJ stent was inserted but patient had recurrent fever and chills. On CECT evaluation authors noticed that DJ stent was outside the upper urinary tract. And then patient was re-explored where authors found $6 \mathrm{~cm}$ stricture of the proximal left ureter. A reconfigured small bowel tube was interposed between inferior calyx and proximal ureteral stumps. This technique offers certain distinct advantages. A short ileal segment is included with the consequent absence of metabolic complications. Yang-Monti Principle is a safer and efficient technique for clinical partial and complete ureteral defects with sustained, good, long-term results. Yang-Monti reconfigured tube seems to be promoted an equally efficient urine transport mechanism that persists unaltered for long periods if patients and potential risks could be well prepared.
\end{abstract}

Keywords: Ileum, Reconstruction, Ureter, Ureteral substitution, Yang-Monti

\section{INTRODUCTION}

Reconstruction of proximal left ureteral defects or stenotic sections with a short simple ileal segment. ${ }^{1,2}$ In the past decade, however, there has been a trend towards using reconfigured small sections of either ileum or colon for reconstruction. ${ }^{3}$ The reconfigurement of bowel segments into tubes of suitable length and diameter was originally described by Yang in 1993 and verified by Monti et al in 1997 as an alternative to using the appendix for continent urinary diversions., ${ }^{4,5}$ The YangMonti technique is helpful when the appendix is too short or is surgically absent or left side proximal ureteral defect. ${ }^{4,5}$ More clinical reports of using this Yang-Monti principle in bridging proximal large ureteral defects are emerging. ${ }^{6,7}$ In this case report, authors used Yang Monti tube in bridging proximal ureteric defect with inferior calycostomy.

\section{CASE REPORT}

A 41-year-old male presented with penetrating retroperitoneal injury with isolated left renal pelvic injury with urinoma. Patient was explored and repair of pelvis injury done with renal capsular Thompson's flap over a 6 Fr double J (DJ) stent. After 6 weeks, retrograde pyelography (RGP) was done and DJ stent removed. Following DJ stent removal patient developed urinoma 
and percutaneous drain is inserted. RGP was done which showed a wall leak and DJ stent was reinserted. After 2 weeks patient developed fever and chills. CECT was done which showed that upper end of DJ stent migrated outside the upper urinary tract indicating pelviuretric junction disruption (Figure 1). Re-exploration was planned and on re-exploration there was dense adhesions enveloping the upper ureter and pelvis. On dissection stricture of proximal ureter was found with compromised vascularity (Figure 2). There was a gap of $6 \mathrm{~cm}$ between the viable ureter and lower pole of kidney so we planned to use ileal segment to bridge the gap as dissection of kidney not possible due to dense adhesion and appendix could not be mobilized to left side (Figure 3). A $2.5 \mathrm{~cm}$ segment of ileum with a suitable blood supply was taken (Figure 3), opened along its anti-mesenteric border and then closed transversely surrounding a 10 Fr urethral catheter using intermittent PDS 4-0 sutures (Figure 4). The diameter of the small intestine determined the length of the newly built tube. Anastomosis of Yang-Monti's tube to the proximal ureteric stump and inferior calyx after placement of 6 Fr DJ stent and PCN insertion and omentum wrapped around Yang-Monti tube (Figure 5 and 6). The patient was discharged on day 6 with PCN and DJ stent in situ and authors are planning to do a nephrostogram on day 21 .

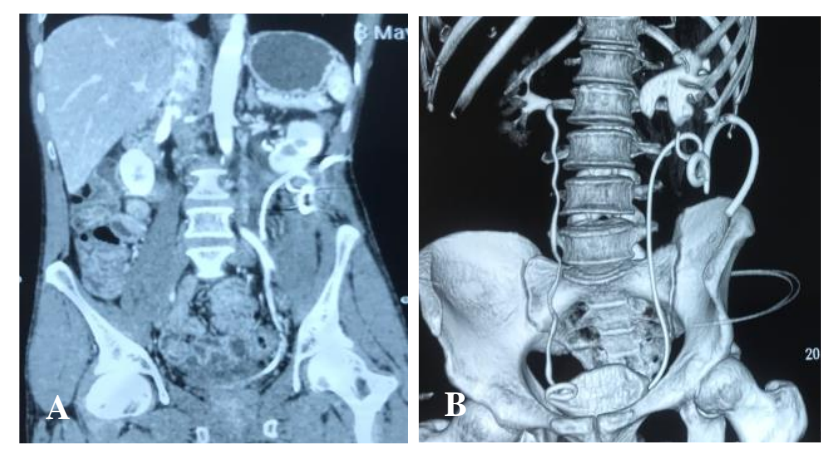

Figure 1: CECT W/A (A) axial and (B) 3D reconstruction film showed that upper end of $\mathrm{DJ}$ stent migrated outside the upper urinary tract indicating pelviureteric junction disruption and percutaneous drain in situ to drain urinoma.

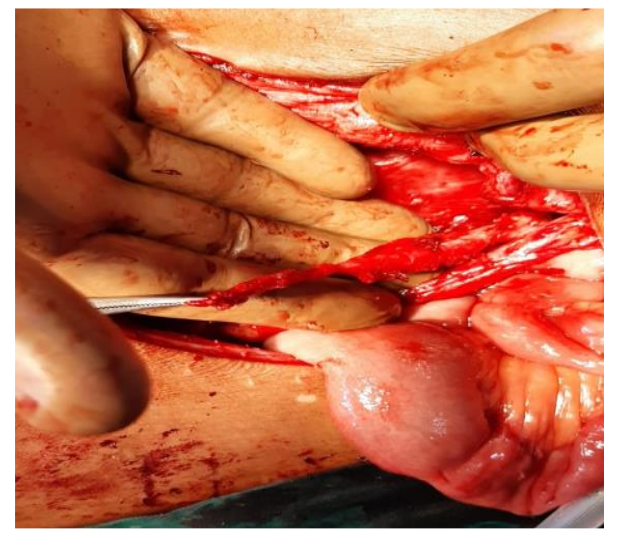

Figure 2: Proximal ureteric stricture with compromised vascularity.

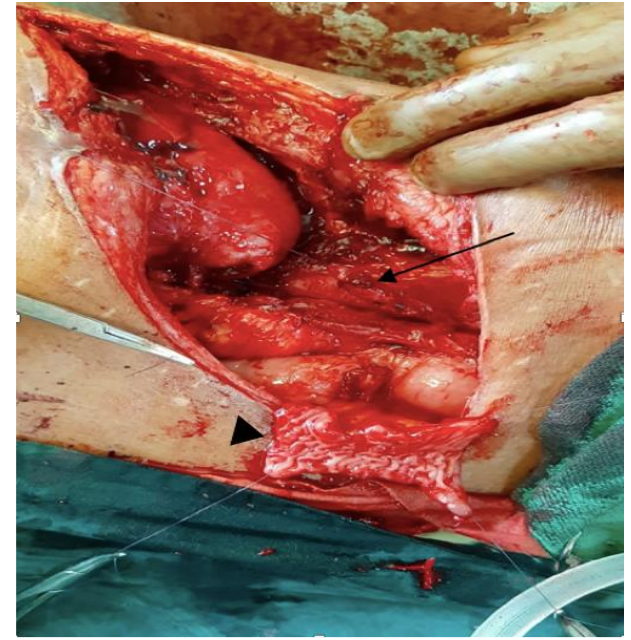

Figure 3: Gap of $6 \mathrm{~cm}$ between the viable ureter (arrow) and lower pole of kidney and a $2.5 \mathrm{~cm}$ segment of ileum with a suitable blood supply (opened at antimesenteric border, arrow head).

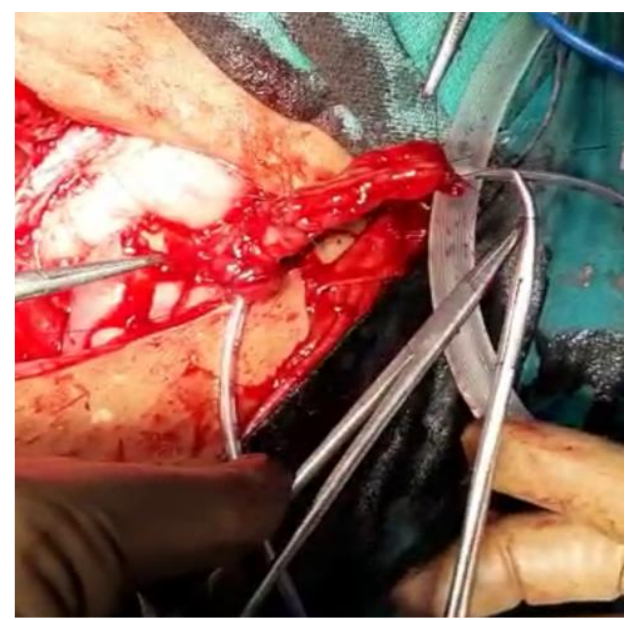

Figure 4: Ileal segment closed transversely surrounding a 10 fr urethral catheter using intermittent PDS 4-0 sutures.

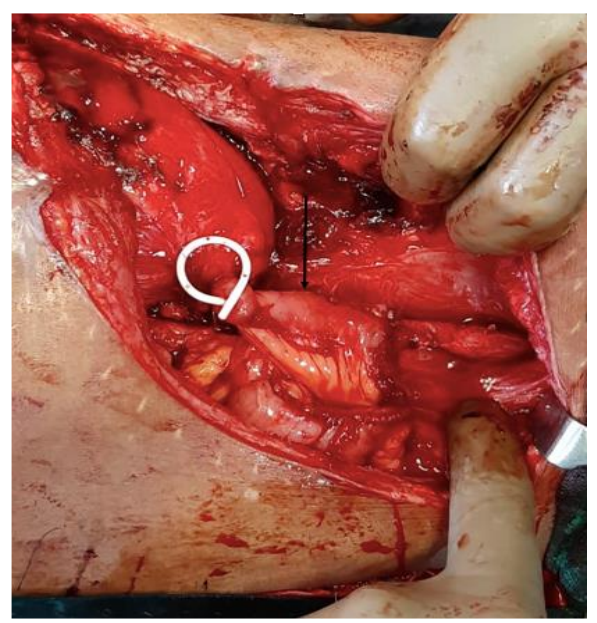

Figure 5: Yang monti tube anastomosed with left proximal ureter (arrow) over DJ stent. 


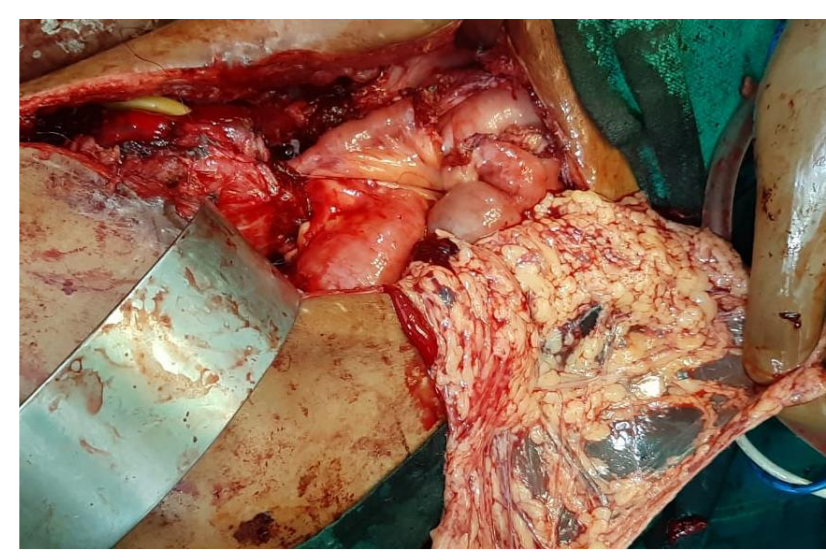

Figure 6: Yang monti tube for inferior ureterocalycostomy and omentum prepared for wrapping (arrow) around young monti tube.

\section{DISCUSSION}

In 1993, Yang was the first to describe two small previously detubularized ileal segments to develop transverse tube in a patient undergoing radical cystectomy. ${ }^{4}$ The merits of the ileal segment are its mobility, small diameter, and constant blood supply. ${ }^{8}$ However, common postoperative risks were urine leakage, peritonitis, colic, strictures and infections. ${ }^{9}$ In addition, drawbacks mostly attributed to the absorbing and secreting characteristics of the involved bowel segments such as hyperchloremic metabolic acidosis and excess mucus production and also to the wide caliber refluxing ileal ureter with subsequent progressive dilatation, functional obstruction and recurrent UTI. ${ }^{10}$ In this case report authors used Yang-Monti tube for inferior ureterocalycostomy to bridge proximal ureteric defect. Authors located ureteric replacement in the retroperitoneum, which is more accordant to the initial physiological characteristics and can decrease the interference in abdomen. ${ }^{8,10}$ The less secretion of succus entericus might be of benefit for maintaining the normal function, efficient urine drainage and fast recovery of the newly reconstructed urinary tracts. ${ }^{11}$ The intermittent suture method was employed in ileouretrocalycosyomy which might be efficient to decrease the occurrence of urine leakage after operation. ${ }^{11}$ However, this procedure needs more evidence.

\section{CONCLUSION}

The Yang-Monti principle for ureteric substitution is a safe technique with an excellent outcome. It uses short ileal segments for reconstruction of an ileal tube of adequate length and optimal caliber that permits easy implantation into inferior calyx. It is not associated with excess mucus production or metabolic abnormalities and offers a durable preservation of renal function without urinary obstruction. In general, care must be taken to avoid urinary leakage, particularly at the proximal anastomosis, which may result in prolonged hospitalization and DJ stenting. The protocol should include frequent follow-up to avoid long-term loss of ileal segment and renal function. This case is rare in which authors used Yang-Monti principle with inferior ureterocalycostomy.

\section{Funding: No funding sources \\ Conflict of interest: None declared \\ Ethical approval: Not required}

\section{REFERENCES}

1. Franke JJ, Smith JA. Surgery of the ureter. In: Walsh PC, Retik AB, Vaughan ED Jr, et al. Eds. Campbells' Urology. $7^{\text {th }}$ ed. Philadelphia: WB Saunders; 1998(3): 3062-3084.

2. Schoeneich G, Winter P, Albers P, Fröhlich G, Müller SC. Management of complete ureteral replacement: experiences and review of the literature. Scand J Urol Nephrol. 1997;31(4):383-8.

3. Boxer RJ, Fritzsche P, Skinner DG, Kaufman JJ, Belt E, Smith RB, et al. Replacement of the ureter by small intestine: clinical application and results of the ileal ureter in 89 patients. J Urol. 1979;121(6):728-31.

4. Yang WH. Yang needle tunneling technique in creating antireflux and continent mechanisms. J Urol. 1993;150(3):830-4.

5. Monti PR, Lara RC, Dutra MA, de Carvalho JR. New techniques for construction of efferent conduits based on the Mitrofanoff principle. Urology. 1997;49(1):112-5.

6. Castellan M, Gosalbez R. Ureteral replacement using the Yang-Monti principle: long-term followup. Urology. 2006;67(3):476-9.

7. Mitrofanoff P. Continent trans-appendicular cystostomy in the treatment of neurological bladders. Chir Pediatr. 1980;21:297-305.

8. Çetinel B, Demirkesen O, Çetinel Ş, Gül Ü, Kiliç N, Solok V. The use of transversely tubularized bowel segment for segmental ureteral replacement. Urol Inter. 2003;71(3):246-50.

9. Narayanaswamy B, Wilcox DT, Cuckow PM, Duffy PG, Ransley PG. The Yang-Monti ileovesicostomy: a problematic channel?. BJU Inter. 2001;87(9):8615 .

10. Maigaard T, Kirkeby HJ. Yang-Monti ileal ureter reconstruction. Scand J Urol. 2015;49(4):313-8.

Cite this article as: Sarma D, Rajeev TP, Ghanghoria A, Barua SK, Bagchi PK, Phukan M. Post traumatic ureteral defect brigded with ileal segment (YangMonti) for inferior ureterocalycostomy: a rare case report with review of literature. Int Surg J 2020;7:8868 . 